\title{
Constraint Mechanism of Reputation Formation Based on Signaling Model in Social Commerce
}

\author{
Shou Zhaoyu ${ }^{1}$, Hu Xiaoli ${ }^{2}$, Zhang Huibing ${ }^{2, a^{*}}$
}

\begin{abstract}
${ }^{1}$ Key Laboratory of Cognitive Radio and Information Processing of the Ministry of Education, Guilin University of Electronic Technology, Guilin, Guangxi, China

${ }^{2}$ Guangxi Key Laboratory of Trusted Software, Guilin University of Electronic Technology, Guilin, Guangxi, China

azhanghuibing@guet.edu.cn
\end{abstract}

Keywords: social commerce; weak ties; Signaling Model; reputation.

Abstract. A typical feature of weak relationship between the users and sellers emerges in social commerce. The seller's reputation is a key element to ensure smooth online transactions in this weak ties environment. This paper constructs a model which integrated Bayes rule and signaling model to analyses the key factors constraining reputation formation. First, to get the reputation constraint factors under separating equilibrium conditions by setting up a model based on the seller's reputation signal between sellers and users' dynamic game process; then, to establish computing model of the seller's reputation in social commerce according to Bayes' theorem; last, analyzing the meaning of constraints to reputation calculation. This research will provide theoretical guidance to reasonable setting of two key elements affecting the seller's reputation.

\section{Introduction}

With the development of e-commerce, especially social commerce, online transaction shows explosive growth ${ }^{1,}$ online shopping has a profound impact on people's production and life. However, both sides of online transaction revealed a typical feature of weak ties: the users know little information about the seller and the commodities, while the seller has all the product information. They are in a serious information imbalance. Thus some sellers use this information imbalance to sell shoddy low-quality merchandise for high-quality products for illegally high profits, disrupting the online trading environment resulting in a growing trust crisis among consumers and sellers, and hindering the improvement of the number and quality of online transactions ${ }^{2}$. Reputation mechanism is considered to be an effective approach to solve the trust crisis in online trading environment.

\section{Related Works}

Present research on business reputation mechanism in social commerce is mainly from two aspects: the reputation relationship and the impact assessment between social network users, and trust network model in social network. Nepal [1] established a trust model integrated popularity trust and engagement trust based on the fundamental relationship between social capital, interactive relationship and social trust, which aimed to enhance the overall community credibility. Han Yi and others analysed the dependent relationship among individuals in social commerce from the perspective of people's social status and the network structure in which they are thus established a model-dependent supportive measure method and supportive structure model, provided the most supportive node calculation method. The model can better characterize the influence source of particular node with better efficiency and scalability ${ }^{[2]}$. Akshay applied Epinions's data set to study the relationship of trust/mistrust and mutual evaluation among users, noting that a single friend's and his corresponding evaluation is in consistence with his friends' circle and their corresponding evaluation ${ }^{[3]}$. Orman represented the trust relationship between users as a conditional probability T(A/B): the subjective probability of user B's service in accordance with user A's interest and goal; at the same time he introduced environmental information factor such as interest intersection, incentive compatibility, ability, and knowledge with Bayesian networks as a basis for reasoning and analysing, 
deduced from the known trust value to the unknown ${ }^{[4]}$. This model has better adaptability with a solid theoretical foundation and relaxing the requirements of many assumptions. In the light of the huge numbers of nodes in social network and the lower trust calculation efficiency based on trust transfer, Jiang used the weak ties in social network and small-world model proposed an algorithm of trust chain discovery based on users' subject field, with this he put forward trust network and trust graph construction technique ${ }^{[5]}$. Zhang formed a model of trust transmitting mechanism on account of factor graph, transformed trust forecast to statistical inference issue, and used this method to achieve trust prediction among social network users ${ }^{[6]}$.

However, the above researches put emphasis on reputation computing method and its elements dimension, ignoring the constraint mechanism of reputation formation and the reputation acting mechanism. Based on the idea of reference [7], this thesis considers reputation as an intangible asset for sellers. Then we analyses the constraint mechanism of reputation formation by modeling reputation forming process and the impact mechanism on both transaction sides in information imbalance environment by reputation-based signaling model.

\section{Dynamic game in social commerce using reputation based Signaling Model}

In social commerce system, in order to ensure the long-term interests of sellers and the sound development of the market as a whole, it should be ensured that high quality $(H Q)$ goods with the production cost of $P C(H Q)$ can be sold at competitive prices $T C(H Q)$; Low quality $(L Q)$ goods with the production cost of $P C(L Q)$ can be sold at inferior prices $T C(L Q)$; and under normal operation of the market environment it should be $P C(H Q)>P C(L Q) 、 T C(H Q)>T C(L Q)$. At the same time, it is also necessary to make $H Q$ goods with better profitability per unit', namely $(T C(H Q)-P C(H Q))>$ $(T C(L Q)-P C(L Q))$. However, the online transactions in social commerce are "weak ties" interactions, the quality of the product $Q$ is clear only to the seller himself; the user has no idea of the true quality before purchase, but he can learn through experiences accumulated that the probability of that goods belonging to $H Q$ is $\theta$, and to $L Q$ is $1-\theta$. If the user can't further distinguish the pros and cons of quality of goods from the seller, then he can only participate the market transaction by average price $(A P)$ on the basis of the probability and $T C(L Q)<A P<T C(H Q)$ or $(T C(H Q)-A P)<(A P-T C(L Q))$. Thus the profitability of $H Q$ goods reduces while of $L Q$ good enhances, namely $(T C(H Q)-P C(H Q))<(T C$ $(L Q)$ - $P C(L Q))$; the market would be in a state of unhealthy competition. To ensure the healthy development of the market, normally the merchandise's quality $Q$ provided can be shown through the sellers' reputation value $R$ in the online trading environment. The user determines whether to accept the commodity price $P(R)$ or to purchase $B\left(B_{0}\right.$ not buy, $B_{1}$ purchase) after observing the seller's reputation. Thus, the reputation value $R$ has become the key signal of quality transferred from the seller to the user; the user identifies the goods quality via the signals and determines the transaction price $P(R)$. Therefore the trust value $R$ becomes the crux of the dynamic game between sellers and users in this asymmetric information state.

To analyse the key role of reputation of online transactions in social commerce environment under asymmetric information, here we take the seller's reputation value as a signal to analyse the dynamic game process via signaling model. First, we introduce natural $\mathrm{N}$ to establish the dynamic game tree of incomplete information between sellers and users. N selects the seller's item type $Q: H Q$ and $L Q$; the user only knows the probability of $H Q$ or $L Q$ goods are $H Q=\theta, L Q=1-\theta$; the seller sends out reputation signal $R\left(R_{g}, R_{m}, R_{b}\right)$ according to the online transaction process. The user determines an acceptable purchase price based on the seller's reputation signal and decides to purchase or not. If he does, the seller's profit is $u_{1}\left(R, B_{1}, Q\right)$; if not, zero. Wherein, $u_{1}\left(R_{g}, B_{1}, H Q\right)=T C(H Q)-P C(H Q)$, $u_{1}\left(R_{m}, B_{1}, H Q\right)=A P-P C(H Q), u_{1}\left(R_{m}, B_{1}, L Q\right)=A P-P C(L Q), u_{1}\left(R_{b}, B_{1}, L Q\right)=T C(L Q)-P C(L Q)$. After repeated games the separation of different sellers' reputation has been achieved, $H Q$ sellers establish good reputation, while $L Q$ sellers form poor reputation.

To ensure a fair market and smooth online transactions, the restraint mechanism between times of repeated games and seller's reputation $R$ needs to establish: it's necessary to form a recognizable 
sellers' reputation value, at the same time the number of repeated games should be as small as possible. That is, $L Q$ goods should get high profits as short as possible under asymmetric information. In this respect, it assumes that after many times of games the seller's recognizable reputation value has been formed, and users can accurately identify the quality of goods and its corresponding transaction price according to this reputation value once it established. In the previous n-th games, since the original value is $R_{0}$ which is difficultly recognizable. After $n+1$-th, the reputation is good to transact with $T C(H Q) ; L Q$ seller's trust value is as bad as to deal with $T C(L Q)$. The dynamic game tree in Figure 1 gives a description of one game process. In essence, the seller forms his own trust value by repeated dynamic games and gets expected benefits. Assume the discount rate is $\delta$, then the net present value of profits of low quality sellers can be expressed as $u_{1}^{a j}\left(\mathrm{R}, \mathrm{B}_{1}, \mathrm{LQ}\right)$ $=\frac{\left(1-B^{n}\right)(A P-P C(L Q))}{1-5}+\frac{B^{n}(T C(L Q)-P C(L Q))}{1-B} ;$ of high quality sellers as $u_{1}^{z j} \quad\left(R, B_{1}, H Q\right)$ $=\frac{\left(1-8^{n}\right)\left(\mathrm{AP}-1-\mathrm{P}_{(\mathrm{C})}(\mathrm{HQ})\right)}{1-6}+\frac{\mathrm{B}^{n}(\mathrm{TC}(\mathrm{HQ})-\mathrm{PC}(\mathrm{HQ}))}{1-6}$. According to the basic principles of market behaviour, it should be ensured that $u_{1}^{z j}\left(\mathrm{R}, \mathrm{B}_{1}, \mathrm{LQ}\right)<u_{1}^{2 j}\left(\mathrm{R}, \mathrm{B}_{1}, \mathrm{HQ}\right)$, namely: $: \frac{\left(1-\delta^{n}\right)(\mathrm{AP}-\mathrm{PC}(\mathrm{LQ}))}{1-6}+\frac{6^{n}(\mathrm{TC}(\mathrm{LQ})-\mathrm{PC}(\mathrm{LQ}))}{1-6}<$ $\frac{\left(1-\delta^{n}\right)(A P-P C(H Q))}{1-6}+\frac{6^{n}(T C(H Q)-P C(H Q))}{1-6}$. Thus we have $\delta^{n}>\frac{P C C H Q)-P C(L Q)}{\operatorname{TC}(H Q)-T C(L Q)}$.

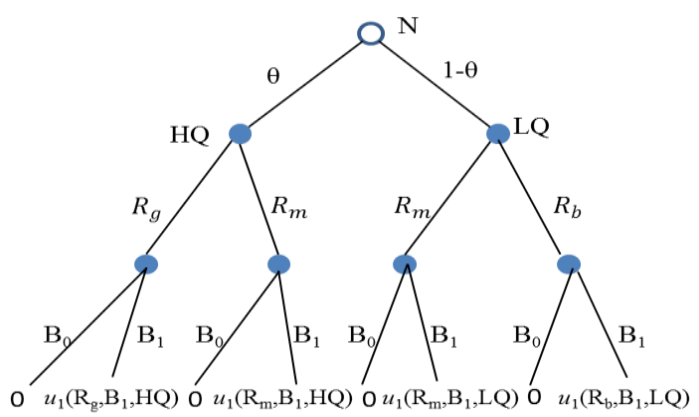

Fig. 1 the Dynamic Game tree based reputation based signaling model.

\section{Evolution mechanism of sellers' reputation probability based on Bayes Rules}

After the text edit has been completed, the paper is ready for the template. Duplicate the template file by using the Save As command, and use the naming convention prescribed by your conference for the name of your paper. In this newly created file, highlight all of the contents and import your prepared text file. You are now ready to style your paper; use the scroll down window on the left of the MS Word Formatting toolbar. We analysed the role of reputation as signals in the commodity trade of social commerce: the only key information which users can get about the goods is the seller's reputation value $R$, accordingly they decide whether to buy this product or not. After users purchase and use the goods they obtain information of the product (assuming that users can perceive the quality and give true evaluation of the goods and they are independent from each other). This information reflects the integrity of the seller: if the evaluation is good, which indicates that the seller is trustworthy, its reputation value rises; on the contrary, decreases. With the increase of the transactions between sellers and users, one can get the seller's reputation value by users' rating. The forming process of this trust value can be described by Bayes Rule.

It's possible for sellers to sell high quality or low quality goods. So they have good reputation $R_{g}$ and bad reputation $R_{b}$, we assume $P\left(R_{g}\right)=p(0 \leq p \leq 1)$, then $P\left(R_{b}\right)=1-p$. We also assume that the trust identification interval is $R_{0}:\left[R_{0} / 2,3 R_{0} / 2\right]$. Within this interval, when the reputation is good, the probability of its selling $H Q$ goods is $P G_{\text {hq }}$, namely $P\left(H Q \mid R_{g}\right)=P G_{\text {hhq }}$, the probability of selling $L Q$ is $P\left(L Q \mid R_{g}\right)=1-P G_{h q}$; if the reputation bad, the probability of its selling $H Q$ goods is $P\left(L Q \mid R_{g}\right)=1-P G_{h q}$, the probability of selling $L Q$ is $P\left(L Q \mid R_{b}\right)=1-P B_{h q}$ (generally, the probability of reputable sellers to sell $H Q$ goods is higher than that of poor reputation ones, therefore $\left.P\left(L Q \mid R_{b}\right)=1-P B_{h q}\right)$. 
Normally, the initial reputation type of a seller is unknown in online trading network environment. In order to ensure fairness we set the reputation neutral $R_{0}: P\left(R_{g}\right)=P\left(R_{b}\right)=0.5$. To the users, it's a priori probability of the seller's reputation. Users can obtain the probability of the merchant's selling $H Q$ or $L Q$ goods according to this probability as the basis for buying decisions. Initially, the first buyer calculates the probability of the merchant's selling $H Q$ or $L Q$ goods according to his initial reputation: in the initial reputation, the probability of selling $H Q$ goods is expected $P(H Q)=P\left(R_{g}\right) * P\left(H Q \mid R_{g}\right)+P\left(R_{b}\right) * P\left(H Q \mid R_{b}\right)=0.5 *\left(P G_{h q}+P B_{h q}\right)$; of selling LQ goods is $P(L Q)=P\left(R_{g}\right) * P\left(L Q \mid R_{g}\right)+P\left(R_{b}\right) * P\left(L Q \mid R_{b}\right)=0.5 *\left(1-P G_{\text {hq }}\right)+0.5 *\left(1-P B_{h q}\right)=$ $1-0.5 *\left(P G_{h q}+P B_{h q}\right)$. The user decides whether to buy with this probability information, if he does, he will observe the quality of the actual goods purchased. Then he can correct the probability of the seller's reputation according to the quality of the goods he observes.

If the purchased goods are $L Q$, then the posterior probability of the seller's poor reputation $P_{1}\left(R_{b} \mid L Q\right)$ is: $P_{1}\left(R_{b} \mid L Q\right)=\frac{P\left(R_{b}\right) * P\left(L Q \mid R_{b}\right)}{\left.P\left(R_{g}\right) * P(L Q) R_{g}\right)+P\left(R_{b}\right) * P\left(L Q \mid R_{b}\right)}=\frac{0.5 *\left(1-P E_{h q}\right)}{0.5 *\left(1-P G_{h q}\right)+0.5 *\left(1-P B_{h q}\right)}=\frac{\left(1-P B_{h q}\right)}{\left(1-P G_{h q}\right)+\left(1-P s_{h q}\right)}$

$$
=\frac{1}{\left(1-P G_{h q}\right) /\left(1-P B_{h q}\right)+1}>0.5
$$

If the purchase is $L Q$ goods, then the posterior probability of the seller's good reputation $P_{1}\left(R_{g} \mid L Q\right)$ is: $P_{1}\left(R_{g} \mid L Q\right)=\frac{L_{\left.P\left(R_{g}\right] * P[L Q] R_{g}\right]}}{\left.P\left(R_{b}\right) * P\left(L Q \mid R_{b}\right)+P\left(R_{g}\right) * P(L Q] R_{g}\right)}=\frac{0.5 *\left(1-P G_{h q}\right)}{0.5 *\left(1-P B_{h q}\right)+0.5 *\left(1-P G_{h q}\right)}$

$$
=\frac{1}{\left(1-P B_{\text {hq }}\right) /\left(1-P G_{h q}\right)+1}<0.5
$$

If the purchase is $H Q$ goods, then the posterior probability of the seller's good reputation $P_{1}\left(R_{g} \mid H Q\right)$ is: $P_{1}\left(R_{g} \mid H Q\right)=\frac{P\left(R_{g}\right] * P\left(H Q \mid R_{g}\right)}{\left.\left.P\left(R_{b}\right) * P(H Q] R_{b}\right)+P\left(R_{g}\right) * P(H Q] R_{g}\right)}=\frac{0.5 * P G_{h q}}{0.5 * P E_{h q}+0.5 * P G_{h q}}$

$$
=\frac{1}{P B_{h q} / P G_{h q}+1}>0.5
$$

Likewise, if the purchase is $H Q$ goods, then the posterior probability of the seller's poor reputation is $P_{1}\left(R_{b} \mid H Q\right)<0.5$.

The second user takes the posterior probability the first user corrected as his prior probability: if it's $H Q$ goods seller, then the second user's prior probability is $P_{2}(g)=P_{1}\left(R_{g} \mid H Q\right)$; $P_{2}(b)=P_{1}\left(R_{b} \mid H Q\right)$; if it's a $L Q$ one, then the second user's prior probability is $P_{2}(g)=P_{1}\left(R_{g} \mid L Q\right)$; $P_{2}(b)=P_{1}\left(R_{b} \mid L Q\right)$. Thus the prior probability of the $n$-th user sees is the posterior probability of $n$ - 1 th user. After $N$ times' transaction, if the purchase is $H Q$ item, the posterior probability for good reputation is $P_{n}\left(R_{g} \mid H Q\right)=1 /\left(1+\left(P B_{h q} / P G_{h q}\right)^{n}\right)$; the posterior probability for bad reputation is $P_{n}\left(R_{b} \mid H Q\right)=1 /\left(1+\left(P G_{h q} / P B_{h q}\right)^{7 \pi}\right)$; similarly, if the purchase is of $L Q$ goods, the posterior probability for good reputation is $P_{n}\left(R_{g} \mid L Q\right)=1 /\left(1+\left(\left(1-P B_{h q}\right) /\left(1-P G_{h q}\right)\right)^{n}\right)$; the posterior probability for bad reputation is $P_{n n}\left(R_{b} \mid L Q\right)=1 /\left(1+\left(\left(1-P G_{h q}\right) /\left(1-P B_{h q}\right)\right)^{n}\right)$.

It can be seen that when the user observes the item's true quality, the seller's reputation changes accordingly: if the sale is of $H Q$ commodity then its trust value rises, if of $L Q$ goods then the value decreases. To obtain the seller's trust value, we need to map the probability value of good or bad reputation into trust value: $R=f(P(g), P(b))$. For the convenience of discussion, we assume that a particular seller only sells $H Q$ or $L Q$ goods, then his reputation value may continuously rise or fall, and the online transaction will gradually grow with the increase of its reputation; on the other hand, if his reputation value reduces to a certain threshold in the future, then users don't trust him anymore. And so there will be no online transactions again. 


\section{Analysis and conclusion}

Reputation is an intangible assets for sellers formed after repeated games between buyers and them. Revealing the game mechanism and constraint elements between sellers and users is an important guarantee to design and achieve a good trust mechanism for online transaction. To simplify discussion, we take sellers who sells high quality goods as an example, and suppose that $f(P(g), P(b))=2 R_{0} P(g)-2 R_{0} P(b)$. At the end of the $n$-th trade, its business reputation is $R=f(P(g), P(b))=f\left(1 /\left(1+\left(P B_{h q} / P G_{h q}\right)^{n}\right), 1 /\left(1+\left(P G_{h q} / P B_{h q}\right)^{n}\right)\right)=$ $2 R_{0}\left(1 /\left(1+\left(P B_{h q} / P G_{h q}\right)^{n}\right)-1 /\left(1+\left(P G_{h q} / P B_{h q}\right)^{n}\right)\right)$

Meanwhile, in order to ensure the market fairness and credibility recognition, assuming that after the $n$-th transaction we claim for $\mathrm{R}>3 R_{0} / 2$, namely $\left(1 /\left(1+\left(P B_{h q} / P G_{h q}\right)^{n}\right)-1 /\left(1+\left(P G_{h q} / P B_{h q}\right)^{n}\right)\right)>3 / 4$ and $\delta^{n}>\frac{\mathrm{PC}(\mathrm{HQ})-\mathrm{PC}(\mathrm{LQ})}{\mathrm{TC}(\mathrm{HQ})-\mathrm{TC}(\mathrm{LQ})}$. we obtain $\mathrm{n}<\log _{\delta} \frac{\mathrm{PC}(\mathrm{HQ})-\mathrm{PC}(\mathrm{LQ})}{\mathrm{TC}(\mathrm{HQ})-\mathrm{TC}(\mathrm{LQ})} ; \quad P B_{h q} / P G_{h q}<\sqrt[n]{1 / 7}$ after solving the inequality above. Thereby two key constraint elements appear in calculating sellers' business reputation: the number of games between users and sellers, and the restrictions to the relations of $P G_{h q}, P B_{h q}$. The two elements reveal the relation of economic interests between sellers and buyers in the forming process of sellers' reputation, and provide the information content transferred by reputation value as signal. It should be noted that the proposed method hereby has better applicability and can be used for other reputation formation as well for trust computing environments.

\section{Acknowledgment}

This research was supported by supported by Education Department of Guangxi (ZD2014049, 2013YB084), Guangxi Natural Science Foundation(2014GXNSFDA118036, 2013GXNSFAA019324, 2013GXNSFBA019267), and the high level of innovation team of Colleges and universities in Guangxi and outstanding scholars program funding.

\section{References}

[1] Nepal, S., Sherchan, W., Paris, C. STrust: A trust model for social networks. 2011 IEEE 10th International Conference on Trust, Security and Privacy in Computing and Communications (TrustCom). (2011)841-846.

[2] Han Yi, Xu Jin, et al. Social Network Supporting Theories. Chinese Journal of Computers. 37(4)(2014) 903-914.

[3] Akshay Patil, Golnaz Ghasemiesfeh, Roozbeh Ebrahimi, Jie Gao. Quantifying Social Influence in Epinions. Proceedings of ASE/IEEE International Conference on Social Computing (SocialCom). (2013)87-92.

[4] Orman, L. V. Bayesian inference in trust networks. ACM Trans. Manage. Inf. Syst. 4(2)(2013)Article 7.

[5] Wenjun Jiang, Guojun Wang, Jie Wu. Generating trusted graphs for trust evaluation in online social networks. Future Generation Computer Systems. 31(2014)48-58.

[6] Richong Zhang, Yongyi Mao.Trust prediction via belief propagation. ACM Trans. Inf. Syst. 32(3) (2014) Article 15.

[7] Tu Chuan qing, Wang Ai hu. The Economic Analysis on the Regional Public Brand of Agricultural Products: A Signaling Game Model Based on Reputation. Journal of Business Economics.253 (11)( 2012)15-23. 